JPAM

\title{
A Conceptual Model of Ambiguity Attitudes for Entrepreneurship Experiments
}

Jacqueline Csonka-Peeren, Brian P. Cozzarin

Department of Management Sciences, University of Waterloo

\section{Author Note}

Jacqueline Csonka-Peeren (iD) http://orcid.org/0000-0002-4863-1835

Brian P. Cozzarin (iD http://orcid.org/0000-0002-4280-7633

We have no conflicts of interest to disclose.

Correspondence concerning this article should be addressed to Jacqueline Csonka-Peeren, Department of Management Sciences, University of Waterloo, 200 University Ave W, Waterloo, ON N2L 3G1, Canada.

Email: jcsonka@uwaterloo.ca 


\begin{abstract}
Ambiguity is central to entrepreneurship phenomena. This paper addresses how ambiguity affects decision making in general, and in entrepreneurship specifically. The work described in this paper relies on robust empirical findings from behavioral economics to construct a conceptual model of factors that influence ambiguity attitudes. The conceptual model includes a novel structure called the ambiguity profile, used to describe an ambiguous situation as perceived by the decision maker. We discuss the applications of this theory for experimental work to understand bias in financial decision making in general, and entrepreneurial financial decision making in particular. Similar or synonymous terminology from various literature is compared to encourage interdisciplinary readership and communication.
\end{abstract}

Keywords: ambiguity, entrepreneurship, bias, entrepreneurial finance, Knightian uncertainty 


\section{A Conceptual Model \\ of Ambiguity Attitudes \\ for Entrepreneurship Experiments}

Entrepreneurs regularly confront situations in which they must make decisions with virtually no information to help inform these decisions. For instance, in making a decision to pursue new opportunities, information that would be helpful such as market size, market growth rate, distribution channels, and funding alternatives, is limited, at best. This missing information creates 'Knightian uncertainty', which is what Knight (1921) described as 'unmeasurable and unquantifiable risk', where risk is comprised of a distribution of both probabilities and outcomes. Understanding decision-making behavior in entrepreneurship therefore includes understanding the nature of Knightian uncertainty and how people respond to it.

In behavioral economics, Knightian uncertainty is called 'ambiguity'. Under condition of ambiguity, outcomes or their likelihoods are not only unknown, but also unknowable. A good deal of empirical research to understand decision-making behavior under condition of ambiguity has been done in behavioral economics. In fact, behavioral economics has an established history of rigorous experiments dating back to the first usage of the concept 'ambiguity' in business literature. The operationalized construct is called 'ambiguity attitudes' and has been validated through repeated experiments both within and outside of behavioral economics. For instance, behavioral decision-making experiments under condition of ambiguity have been repeated in neuroscience, helping to explain the neural mechanisms involved in determining ambiguity attitudes, e.g., Krain et al. (2006).

How does ambiguity affect decision-making behavior in general, and specifically in entrepreneurship? To begin to address this question, two needs arise. First, there is a need to understand what is already known about how ambiguity affects decision-making behavior and, second, there is a need to synthesize this knowledge in a format that can be helpful to study how ambiguity affects decision-making behavior in entrepreneurship in particular. This paper addresses these two needs by constructing a conceptual model of experimental variables to consider for future study of ambiguity attitudes in a context that is representative of entrepreneurship. These variables are identified from the findings of previous research. This work relies on empirical evidence of what is known about behavior under condition of ambiguity studied in behavioral economics. Specifically, this work relies on empirical evidence of ambiguity attitudes during financial decision-making under conditions of financial ambiguity. In synthesizing these findings, a third need arises to clarify the nature of ambiguity, namely to describe the nature of ambiguity endogenous to the conceptual model. This paper addresses this need by introducing a novel structure in the conceptual model called the 'ambiguity profile'. The goal of this paper is to contribute to theory building about decision-making behavior under condition of ambiguity in general, and in entrepreneurship specifically. In theorizing, this paper provides three contributions. First, this paper introduces a new structure called the ambiguity profile, which is a multi-dimensional array of elements that collectively describe an ambiguous situation as perceived by the decision maker. Each of these elements have been found to affect the ambiguity attitudes of a decision maker. The elements of the ambiguity profile include the following: whether the outcome could have gain, loss, or both; the relative magnitude of the outcome; the width of the range of the magnitude of the outcome; the relative probability of the outcome; the width of the range of the probability of the outcome, and the time horizon to the 


\section{AMBIGUITY AND ENTREPRENEURSHIP}

outcome. This ambiguity profile is intended to provide two advantages: to provide researchers with a means to completely describe the combination of elements that are manipulated in an experimental study and to facilitate the comparison of manipulations across studies.

The elements that are included in the ambiguity profile emerged during our literature review of the most reputable empirical research, and are comprised of the variables that were manipulated in previous research studies on ambiguity attitudes towards financial risk, financial uncertainty and financial ambiguity (collectively, financial likelihood). While the findings in each of these three research areas overlap, some differences are predicted. One reason for this is that the human brain is known to process [financial] risk and ambiguity differently (Hsu et al., 2005; Rustichini et al., 2005; Smith et al., 2002).

Second, this paper contributes a coherent conceptual model of all the factors that are known to affect ambiguity attitudes - including the elements of the ambiguity profile. This conceptual model is useful in two ways. First, it can be used to completely describe the experimental variables that were controlled, manipulated and measured in prior research, and the relationship between these variables. Second, it can be used to design further experiments. This paper describes how further experiments could help fill gaps in understanding ambiguity attitudes in an entrepreneurial context. The conceptual model can be used to choose experimental variables to control and variables to manipulate that, collectively, best represent an ambiguous entrepreneurial context.

Third, this paper contributes to understanding theoretical concepts by disambiguating potentially confusing terminology from various literature. Throughout this article, we identify terminology that is similar or has been used synonymously with the terminology used in this paper, and clarify its usage in each case. The intention is to facilitate understanding of the terminology used here and reduce potential confusion for the entrepreneurship researcher who may be familiar with similar terminology used differently across entrepreneurship, behavioral economics, project management and corporate finance.

This paper begins by describing the gap in literature about financial decision-making behavior under condition of ambiguity. Next, it describes the conceptual model of factors affecting ambiguity attitudes, including the ambiguity profile, with a detailed explanation of each variable included in the model. Where applicable, the variables are interpreted in the context of entrepreneurship. This is followed by a description of factors that are known to influence risk attitudes and why those factors might need to be included in the conceptual model. Before concluding, this paper discusses how these novel items could be used by researchers who wish to further our academic understanding of how people behave under condition of ambiguity, and by entrepreneurship researchers in particular. Future theoretical and empirical work is suggested. To begin, we describe how there is a dearth of empirical evidence about how people behave under condition of ambiguity. While this is true in general, it is also true in behavioral economics despite its established history of empirical work in decision making. 


\section{A Gap in Research on Ambiguity}

It has long been acknowledged that decisions made under conditions of risk or uncertainty are central to entrepreneurship phenomena (Knight, 1921; McMullen \& Shepherd, 2006). The terms risk and uncertainty have different meanings. Risk is defined as a set of known outcome probabilities. For instance, a fair coin flip is a risky scenario where the coin has a 50\% probability of landing on either side. Uncertainty, on the other hand, refers to objectively unknown outcome distributions (even if these may be subjectively estimable).

Ambiguity takes the concept of uncertainty further, describing conditions under which outcomes and their likelihoods are not only unknown (objectively and subjectively to the decision maker), but also unknowable (not even subjectively by the decision maker). This definition is consistent with how the term 'ambiguity' is used in behavioral economics literature, including in decision sciences literature (Du \& Budescu, 2005; Liu \& Öncüler, 2017; Onay et al., 2013). In behavioral economics, ambiguity has broadly been described as perceived 'missing information' (Frisch \& Baron, 1988). Within the scope our research, the nature of this missing information is limited to 'Knightian uncertainty', which is what Knight (1921) described as 'unmeasurable and unquantifiable risk', where risk is comprised of a distribution of both probabilities and outcomes.

While a great deal of research has looked at human decision-making behavior under conditions of financial risk, relatively little considers decision-making behavior under conditions of financial ambiguity. Platt and Huettel (2008) also remark on this. For instance, while our literature search uncovered over 20,000 journal articles related to financial risk, fewer than 350 were found for financial ambiguity. This number reduced to fewer than 40 when the search was narrowed to decision-making. None of those articles describe a complete model of the relationships that have been found to impact decision-making behavior under condition of ambiguity. A model of these relationships could help inform decision-making behavior under condition of Knightian uncertainty. We turned our attention to assembling a conceptual model, relying on robust empirical findings.

To find relevant literature, we performed a new search for empirical or experimental findings in decision-making behavior under condition of ambiguity (not just financial). We considered all articles from top journals that were relevant or highly cited, articles that those articles cited or were cited by those articles. We also considered additional sources that were recommended by scholars in decision making, neuroeconomics, and entrepreneurship. Briefly, the most broadly used measure of decision-making behavior under condition of ambiguity is called 'ambiguity attitudes'. This is distinct from the measure used to measure decision-making behavior under condition of risk, which is called 'risk attitudes'. These measures are used in multiple disciplines including decision sciences (e.g., Du \& Budescu, 2005), neuroeconomics (e.g., Huettel et al., 2006), and entrepreneurship (e.g., Lévesque \& Schade (2005)).

The large difference in the quantity of published research related to financial risk as compared to financial ambiguity is important for two reasons. First, experiments repeatedly find no correlation between risk attitudes and ambiguity attitudes (Camerer \& Weber, 1992). Therefore, experimental results of risk attitudes do not inform ambiguity attitudes. Second, risk 
and ambiguity are processed differently by the human brain. Neuroscience research has consistently and repeatedly provided evidence that the brain processes risk, uncertainty, and ambiguity differently. For instance, 'risky gambles' (i.e., gambles involving no ambiguous choice but at least one risky choice) activate more portions of the brain associated with emotions (Hsu et al., 2005; Platt \& Huettel, 2008; Sturm et al., 2016), whereas 'ambiguous gambles' (i.e., gambles involving at least one ambiguous choice) activate more portions of the brain responsible for controlling emotions (Krain et al., 2006; Tanaka et al., 2015), such as inhibition of impulsive behavior (Huettel et al., 2006). Because risk and ambiguity are processed differently by the brain, the neural pathways involved in their mechanism of action will be different; in other words, the causal variables affecting decision-making behavior under condition of risk are not anticipated to be the same as those affecting decision-making behavior under condition of ambiguity.

In sum, Knightian uncertainty is comprised of ambiguity and relevant to the context of entrepreneurial decision-making. Researchers have recently recommended that future research on entrepreneurial behavior consider theorizing and measuring ambiguity (Zhang \& Cueto, 2017). This paper contributes to entrepreneurship theory to address this gap.

\section{Conceptual Model}

The literature described in the sections to follow detail how several variables are expected to impact ambiguity attitudes of entrepreneurs and how ambiguity attitudes, in turn, impact ambiguity preferences. These variables and their relationship are illustrated in Figure 1 and described below.

\section{Figure 1}

Conceptual Model of Experimental Variables to Consider When Studying Ambiguity Attitudes in a Context That is Representative of Entrepreneurship

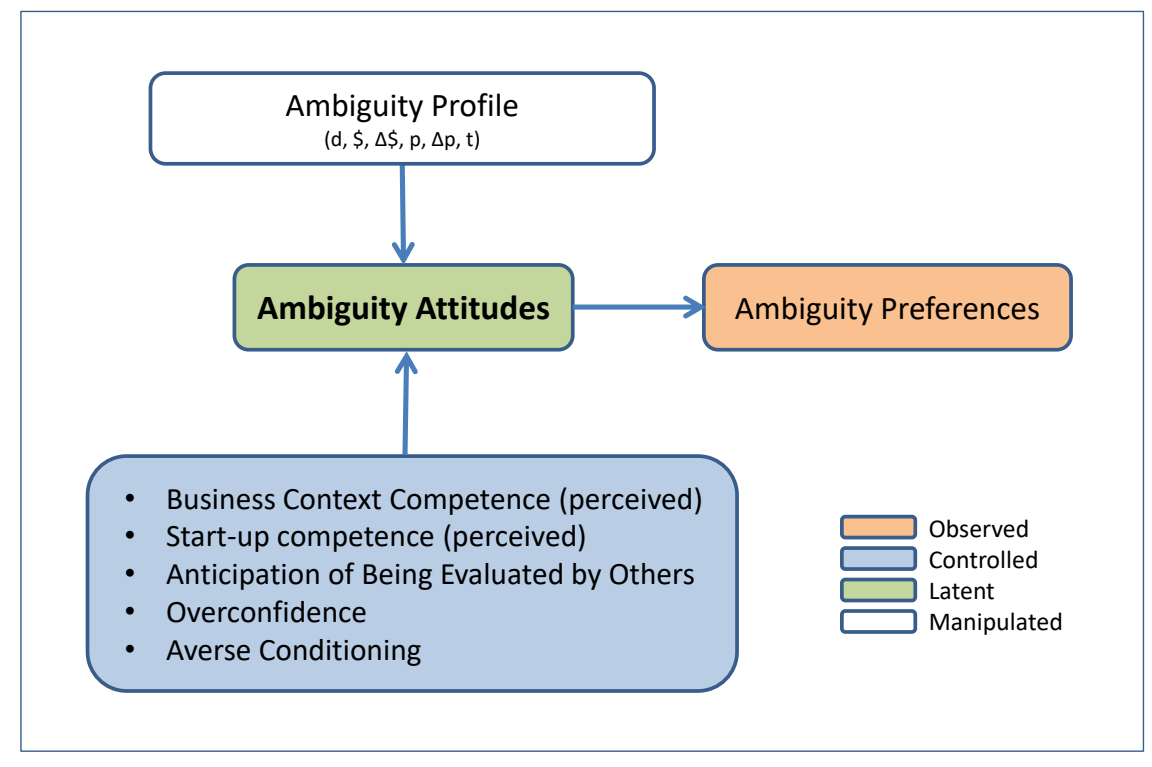

Copyright (C) Institute of Behavioral and Applied Management. All Rights Reserved. 


\section{Ambiguity Attitudes}

Several terms are used interchangeably in the literature to denote ambiguity attitudes, namely 'ambiguity aversion' (e.g., Liu \& Öncüler (2017)) and 'attitudes toward ambiguity' (Onay et al., 2013). By convention, the terms 'ambiguity attitudes' and 'ambiguity preferences' are pluralized in literature.

Relatively few studies have considered financial ambiguity attitudes; however, in those studies, two types of experimental tasks (protocols) are widely used, namely certainty equivalent task and pairwise choice task. Under a certainty equivalent protocol, ambiguity attitudes is calculated from a 'certainty equivalent' measurement, and under a pairwise choice protocol, ambiguity attitudes is deduced from 'ambiguity preferences' measurement.

In a certainty equivalency task, subjects reveal the amount of payoff they would require to be indifferent between this amount of payoff and a given gamble, and ambiguity attitudes is calculated from this payoff (subjective value). This is different from the more widely applied pairwise choice protocol.

Under a pairwise choice protocol, ambiguity attitudes is deduced from ambiguity preferences, which is observed (Du \& Budescu, 2005; Liu \& Öncüler, 2017; Onay et al., 2013). This protocol requires participants to choose their preference between two fictional alternatives that are the same except for the variable of interest, which is manipulated. Ambiguity associated with outcomes is manipulated separately from that of probabilities. Subjects may also be given a choice of 'indifference' (equal preference). Ambiguity attitudes is made evident by a subject's preferred pairwise choice between two 'vague' alternatives differing only in Expected Value (EV). Specifically, ambiguity attitudes is determined by comparing the EV of the choice to that of the un-chosen alternative. In these experiments, outcomes and probabilities are described with a range of values that are known and actual numbers are provided; consequently, a value for EV can be calculated. This type of ambiguity, with known range of values with actual numbers provided, has been referred to as 'partial ambiguity' (Rustichini et al., 2005) and the values delineating a range is described as 'vague' or 'imprecise' (e.g., Onay et al., 2013). The pairwise choice protocol can be used to reveal tendencies in a population across a variable of interest. For instance, business students of the senior- and graduate-level have been shown to display a tendency toward preference for information about vague probabilities over vague outcomes for gambles that are resolved in the near-term (Onay et al., 2013).

Our extensive literature review reveals several variables that are expected to impact on ambiguity attitudes, namely specific elements of the ambiguity profile, business context competence (perceived), start-up competence (perceived), anticipation of being evaluated by others, overconfidence, and averse conditioning. These variables are described in turn below.

\section{Ambiguity Profile}

Collectively, researchers have empirically found that ambiguity preferences (e.g., where ambiguity attitudes is deduced from pairwise choice) and ambiguity attitudes (e.g., where ambiguity attitudes is calculated from certainty equivalent) are affected by several variables. We have assembled some of these variables into what we have labeled the ambiguity profile. The 
ambiguity profile is a combination of elements that describe an ambiguous situation. The elements (variables) of the ambiguity profile are denoted in Figure 1. These elements are domain (d), magnitude of outcome (\$), width of the range of outcome $(\Delta \mathbf{S})$, magnitude of probability (p), width of the range of probability $(\Delta \mathbf{p})$, and time horizon $(\mathbf{t})$. The effects of these elements can be described as follows: reference-domain effects arising from an outcome that is anticipated to fall in the domain of either a gain or a loss (i.e., in $\mathbf{d}$ ); magnitude effects arising from the anticipated magnitude of an outcome (i.e., from \$) or for the probability (i.e., from p); range effects arising from manipulation of the width of the range of anticipated magnitudes of an outcome (i.e., of $\Delta \mathbf{\$}$ ) or from the width of the range of probability (i.e., of $\Delta \mathbf{p}$ ), and temporal effects arising from an outcome that is anticipated to occur after a time horizon that is either nearer- or longer-term (i.e., after $\mathbf{t}$ ).

As discussed, ambiguity attitudes is deduced from ambiguity preferences; therefore, ambiguity attitudes will precede ambiguity preferences in a causal chain and there is a direct relationship between the two measures. Manipulating elements of the ambiguity profile will affect both ambiguity attitudes and ambiguity preferences; however, ambiguity profile will have a direct effect on ambiguity attitudes and an effect on ambiguity preferences via the causal link from ambiguity attitudes. Some studies have reported their findings in terms of effects on ambiguity attitudes and other studies have reported their findings in terms of effects on ambiguity preferences. The two sections below describe findings by type of reporting.

\section{Ambiguity Profile Effects on Ambiguity Attitudes}

Some studies have reported their findings in terms of ambiguity attitudes. From those studies, the elements of the ambiguity profile reported to influence ambiguity attitudes are domain (d), magnitude of outcome (\$), magnitude of probability (p), and time horizon (t). These findings, and how they relate to an entrepreneurial context, are described below.

\section{Domain}

A study of insurance premiums found that clients are willing to pay higher premiums for certainty when there is either ambiguity regarding the probability of a particular loss event occurring and/or uncertainty about the magnitude of the resulting loss. This study was performed for the loss domain. (Kunreuther et al., 1995). Entrepreneurial events have the possibility of high potential loss and high potential gain, e.g., "market segment A could help grow my company but, if it fails, I will be bankrupt." This type of mixed domain is representative of an entrepreneurial context and has not been studied experimentally in prior literature. It is a gap in experimental entrepreneurship.

\section{Magnitudes}

In both probability and outcome, subjects display ambiguity seeking when performing a certainty equivalency task under a condition of unlikely (i.e., low probability) gains or likely (i.e., high probability) losses (Budescu et al., 2002). This is fortunate for entrepreneurship activity because low probability gains and high probability losses are representative of an entrepreneurial context; it implies behavior that seeks Knightian uncertainty. Additionally, in a repeated pairwise choice task, Liu and Öncüler (2017) ran experiments in the gain domain and 
found that individuals display greater ambiguity aversion for high probabilities than for low probabilities when there was no time horizon (present time).

\section{Time Horizon}

Temporal effects (timing of consequences, i.e., near- vs. long- term) reportedly have a significant influence on ambiguity preferences and, by deduction, on ambiguity attitudes. Specifically, subjects are less averse to imprecise probability and more seeking for imprecise outcome when potential gains would be realized in future as compared to in present (Liu \& Öncüler, 2017; Onay et al., 2013). Onay et al. (2013) found this attitude to be more prominent when both probability and outcome were imprecise. As discussed, aversion was determined relative to EV. This finding has impact in an entrepreneurial context. For instance, the amount of precision provided is anticipated to influence financing behavior, e.g., "should I take this equity offer (near term, more precision) or wait for a better one (long-term, less precision)?" This has not been studied in prior experimental research and constitute a gap in experimental entrepreneurship.

\section{Ambiguity Profile Effects on Ambiguity Preferences}

Some relevant studies have reported their findings in terms of ambiguity preferences. Collectively, those studies point to three elements of the ambiguity profile that influence ambiguity preferences. These elements are domain $(\mathbf{d})$, time horizon $(\mathbf{t})$, and width of the range of probability $(\Delta \mathbf{p})$. These findings, and how they relate to an entrepreneurial context, are described below.

\section{Domain}

In the loss domain, research reports an even split between subjects who prefer more information about probability or outcome when presented with a pairwise choice. (Schoemaker, 1989, Table 2) However, as magnitude of potential losses increased, subjects desired more information about outcome (magnitude of loss) (Schoemaker, 1989). This could explain the results of Du \& Budescu (2005) who found a clear split in the loss domain, where more subjects were willing to allocate funds to acquire more precision about the magnitude of outcome than probability. All these findings contrast with results in the gain domain, where most subjects want more information about probability over outcome. (Schoemaker, 1989, p.47 Table 2)

\section{Time Horizon}

Subjects prefer a future prospect that is vague in both probability and outcome than one that is vague in only one of those dimensions. (Onay et al., 2013). In this experiment, subjects' preference was determined through pairwise choice.

This time horizon effect implies that highly ambiguous entrepreneurial ventures may be have a higher valuation when their forecasts are vague in both probability and outcome than when these are more precise in probability or outcome. At the same time, the domain effects described above are anticipated to reward some precision, for instance when large losses are at 
stake. This combination of time horizon and domain effects has not been studied experimentally, and is relevant to an entrepreneurial context. This is a gap in experimental entrepreneurship.

\section{Range}

As the width of the probability range narrows, the value of information on probabilities is lessened (Schoemaker, 1989, p.49 Figure 3). We believe an alternative explanation to a range effect exists for this change in preference; in Schoemaker's survey questions about this, not only was the width of the range of probability narrowed to $40 \%$ from $100 \%$ but also the expected value of probability (given that subjects were told that distribution was uniform) was also decreased to $20 \%$ from $50 \%$. As discussed above, ambiguity attitudes are affected by magnitude of probability. In particular, ambiguity aversion diminishes (and ambiguity seeking increases) at low probability. This in turn can affect ambiguity preferences such that information about probabilities would be less valued. This range effect, in combination with the domain and time horizon effects, has implications in the valuation of highly ambiguous entrepreneurial ventures. This gap in understand the range effect, and its interaction effects with domain and time horizon, could be further explored in the context of entrepreneurship.

No study has explicitly looked at the effect on ambiguity attitudes of a combination of characteristics representative of an entrepreneurial context. From the description of effects of factors above, we know that interaction effects between factors is possible. Consequently, it is reasonable to anticipate that ambiguity attitudes in an entrepreneurial context will differ that those that have been studied in literature, and this constitutes a gap in experimental entrepreneurship.

In sum, the ambiguity profile describes an ambiguity situation. Elements of the ambiguity profile influence ambiguity attitudes, which in turn influence ambiguity preferences. The conceptual model of Figure 1 includes both a direct link between ambiguity profile and ambiguity attitudes and the causal link between ambiguity attitudes and ambiguity preferences.

\section{Other Factors (Ambiguity Attitudes-related)}

Researchers have empirically found that ambiguity attitudes are affected by several variables other than those that describe an ambiguous situation (those included in the ambiguity profile). These other factors are related to the characteristics of the individual decision maker other than their perception of the ambiguous situation. The following factors are included in Figure 1: perceived business context competence, perceived start-up competence, anticipation of being evaluated by others, overconfidence, and averse conditioning. These factors are addressed in turn below.

\section{Business-Context Competence (Perceived)}

Entrepreneurs operating in uncertain environments and who have more industry experience have been shown to demonstrate more accurate, less biased forecasting performance (Cassar, 2014). Heath and Tversky (1991) noted that context-related competence seems to make ambiguity aversion change to ambiguity seeking. 


\section{Start-up Competence (Perceived)}

People are less likely to make investment choices in geographically distant locations. An explanation is that people perceive themselves as less knowledgeable about distant investments as compared to local ones (Trautmann et al., 2008). Prior research has expected perceived competence to have an influence on ambiguity attitudes (e.g., Onay et al., 2013). In the context entrepreneurship, we specify this as start-up competence.

\section{Anticipation of Being Evaluated by Others}

An anticipation of being evaluated by others is expected to affect ambiguity preferences because Curley et al. (1986) describes how a decision maker will make a choice that they perceive will be the most justifiable to others. In the context of entrepreneurship, the judgment of co-founders, investors, employees and other stakeholders are anticipated to influence ambiguity attitudes.

\section{Overconfidence}

Deligonul et al. (2008) suggest that entrepreneurs tend to dismiss possible future performance states as they enter new markets, and this can contribute to venture failure. Shepherd et al. (2015) have attributed this tendency to entrepreneur overconfidence. This overconfidence is what Camerer et al. (2005) terms 'wishful thinking'. Hogarth and Karelaia (2012) provide an alternative explanation for this excess entry, namely judgement fallibility. Judgement fallibility would be exogenous to our model. While these research groups provide theoretical support for their propositions, none has tested these propositions empirically. We have decided to include this variable in the conceptual model and recommend that worthwhile future work could include developing a proposition to test for its relevance.

\section{Averse Conditioning}

Empirical study suggests that risk aversion conditioning can explain risk avoidance in gambling (Brunborg et al., 2010). Strong evidence supports the key role the amygdala plays in this type of fear learning (Ledoux, 1996). Moreover, the amygdala has a critical role in the context of aversion conditioning to ambiguity - or missing information - which has been done in the field of taste conditioning (St Andre \& Reilly, 2007). Thus, it seems possible that negative aversion conditioning from a bad entrepreneurial experience can have a negative (aversion) effect on ambiguity attitudes, although there is no evidence to suggest the same mechanism can have a reverse (seeking) effect from positive entrepreneurial experience.

In sum, researchers have found factors affecting ambiguity attitudes that are related to the characteristics of the decision maker other than their perception of the ambiguous situation. These have been included in Figure 1.

\section{Factors not Included in the Model (Risk Attitudes-related)}

Both risk attitudes (e.g., risk aversion) and ambiguity attitudes (e.g., ambiguity aversion) are associated with risky decision-making; however, ambiguity attitudes is distinct from risk 
attitudes and is measured separately from risk attitudes. For instance, a person can be risk averse and ambiguity seeking.

Repeatedly, experiments have found no correlation between risk attitudes and ambiguity attitudes (Camerer \& Weber, 1992). However, this does not preclude a possibility that factors known to affect risk attitudes might also affect ambiguity attitudes. At the time of writing, we have yet to find literature on a direct link between ambiguity or uncertainty and the following three factors that are prevalently mentioned in literature associated with risk attitudes: business context, affordable loss, and affect. However, neuroscience reveals that there is much overlap in brain activation by factors affecting risk attitudes and factors affecting ambiguity attitudes (Blankenstein et al., 2017). The impact of any of the three factors on ambiguity attitudes would need to be verified empirically before they could be added to the conceptual model.

\title{
Business Context
}

Business context refers to the attributes of a business situation other than probabilities or outcomes. Schwarzkopf (2006) surveyed 224 business students across situational risk characteristic variables, including how controllable a situation is and how many people are affected by an outcome, and found that business context factors affected participants' risk perceptions of a situation.

\section{Affordable Loss}

Affordable loss relates to how much an entrepreneur feels they can afford to lose and whether they feel they have sufficient resources (relative to an aspired level). In a simulation study, March and Shapira (1992) found that the amount of accumulated resources (relative to a perceived 'survival point' and/or desired 'aspiration level') influence risky choice behavior in decision makers. Dew et al. (2009) rely on behavioral economic theory to predict that individuals who perceive a higher affordable loss are more likely to make the risky choice of pursuing an entrepreneurial career. These researchers also developed propositions related to the affordable loss heuristic that was borne of empirical studies of entrepreneurial expertise (Sarasvathy, 2001). The salience of loss is in accordance to the risk construct proposed in the often-cited book by Yates and Stone (1992). This risk construct is based on three underlying considerations: potential losses, the significance of those losses, and the uncertainty of those losses. Forlani and Mullins (2000) also proposes that new venture risk is framed in terms of potential losses, and not in terms of probabilities, and base this in part on empirical studies by Shapira (1995).

\begin{abstract}
Affect
Emotions affect perceptions of risk in ways that can help explain risk-seeking and riskaversion (Caplin \& Leahy, 2001). Lerner \& Keltner (2000) demonstrated that fearful people are more pessimistic in their estimates of risk and make more risk-averse choices, and angry people are more optimistic in their estimates and make more risk-seeking choices. In a highly-cited book, LeDoux (1996) writes, 'While conscious control over emotions is weak, emotions can flood consciousness. This is so because the wiring of the brain at this point in our evolutionary history is such that connections from the emotional systems to the cognitive systems are stronger than connections from the cognitive systems to the emotional systems.' One specific fear that
\end{abstract}


appears to be associated with risky decision-making in entrepreneurship is the fear of failure (Kollmann et al., 2017). Moreover, these researchers empirically found a relationship between affordable loss and fear of failure: When nascent entrepreneurs were confronted with financial obstacles (i.e., obstacles related to affordable loss), they had a reduced likelihood of exploiting that business opportunity (i.e., taking a gamble), and this was mediated by fear of failure. This study also found these entrepreneurs to have evaluated a lower perceived magnitude of gain for that business opportunity.

In sum, factors affecting risk attitudes may also affect ambiguity attitudes; however, the impact of any of the three factors above on ambiguity attitudes would need to be verified empirically before they could be added to the conceptual model of Figure 1.

\section{Discussion}

The goal of this paper was to theorize about how people behave under condition of ambiguity, a condition that is relevant to the entrepreneurial context. The theoretical contributions of this paper rely on empirical findings in behavioral economics, which has a long history of rigorous experimentation using a validated construct of ambiguity attitudes. While a great deal of empirical study has been done in behavioral economics to study risk attitudes, comparatively less has been done for ambiguity attitudes. This is an important gap because literature reveals that notwithstanding an entrepreneur's attitude to risk, their attitude to ambiguity affects will affect their entrepreneurial behaviour. The lack of research on ambiguity attitudes in general, and in entrepreneurship specifically, provides a greenfield opportunity for decision making and entrepreneurship research.

Decision sciences and neuroscience have advanced scholarly understanding of ambiguity attitudes, at a deep cognitive level. This understanding is underutilized in entrepreneurship, as evident from our literature review, and developing such an understanding answers a call from entrepreneurship research and practice (Davis et al., 2016; Bacigalupo et al., 2016; OECD \& EC, 2015). This paper contributes to theory about attitude to ambiguity at the individual unit of analysis. While the behavioral economics approach to this theorizing has strong merits, it does not preclude alternative approaches, such as an affect-based approach (e.g., Grégoire et al., 2015). Future work could be done to theorize based on other approaches.

\section{Designing Experiments in Entrepreneurship}

Two novel theoretical contributions emerge from this work, and both are helpful in designing experiments that address the above-mentioned gap in entrepreneurship research described by Zhang and Cueto (2017). The first contribution is a conceptual model describing the experimental variables and the relationships between them that should be considered when studying ambiguity attitudes in a context that is representative of entrepreneurship. This contribution has applications in entrepreneurship, particularly in experimental design and survey design. The conceptual model identifies several variables that should be controlled for an experiment to study ambiguity attitudes, namely business context competence (perceived), startup competence (perceived), averse conditioning, overconfidence, and anticipation of being evaluated by others. For instance, to control for these variables in a study about nascent entrepreneurs, the researcher could restrict their population sample to senior level and graduate 
entrepreneurship students from the same upper-level class in entrepreneurship so they could be reasonably be anticipated to share a similar level of entrepreneurship training and work experience (i.e., no entrepreneurial experience outside of a campus business incubator ecosystem). As such, the sample could reasonably be considered homogeneous in both subjects' perceived business context competence and subjects' perceived start-up competence. This choice of population would also help control for subjects' averse conditioning because students such a sample are anticipated to be less likely to yet have had a bad (or good) experience with entrepreneurship. Additionally, sampling students who have followed similar experientiallygrounded entrepreneurship training might help additionally control for overconfidence. By virtue of their status as students, all of those in a sample would be reasonably expected to have similar level of anticipation of being evaluated by others. Any anticipation (or fear) of being evaluated could also be controlled (mitigated) by explaining to survey participants that they there are "no wrong answers' and that results shall be anonymized. While there are many good reasons why students are considered representative of nascent entrepreneurs (Hsu et al., 2017), the ability to control for many of the variables influencing ambiguity attitudes is another.

The second contribution is a structure called the ambiguity profile, which is comprised of elements that influence ambiguity attitudes and collectively describe an ambiguous situation as perceived by the decision maker. The ambiguity profile is comprised of those elements (variables) a researcher could chose from to manipulate the entrepreneurial condition in an experimental setting.

\section{Interdisciplinary Knowledge}

Risky decision-making that includes ambiguity is not restricted to entrepreneurship. This conceptual model can be used to facilitate interdisciplinary communication to advance a collective understanding of how people behave under condition of ambiguity. This paper contributes to facilitating interdisciplinary communications by disambiguating some terminology across disciplines. This could contribute to future theory building in at least two other academic disciplines. For one, it could contribute to theory building in the discipline of project management such as new product development (NPD). In new product development (NPD), the term 'equivocal uncertainty of probability distributions' can be used to describe the type of ambiguity described in this paper (Camerer \& Weber, 1992; Galbraith, 1973; Galbraith, 1974). Project risk management practices developed for NPD could be explored to determine what might be applied to entrepreneurship. Suggestions by Forlani and Mullins (2000) include risk analysis, qualitative and quantitative market research methodologies, and critical assumption planning of Sykes and Dunham (1995). Research in NPD suggests that front-end activity that reduces ambiguity leads to more successful innovation commercialization (Frishammar et al.,2011). In a start-up, the NPD process coincides with the start of the company's operations; in other words, at the founding of a new venture, NPD begins. Therefore, it is likely that at least some of what is known or learned in the context of entrepreneurship might be generalizable to NPD and vice versa.

For another, it could contribute to theory building in the discipline of corporate finance, namely in financial risk management, where ambiguity is called 'unknowable risks' (Diebold et al., c2010). Financial forecasting for an innovative new venture requires guessing about the future, where this guessing is based on limited (or no) information. Knowledge gained from 


\section{AMBIGUITY AND ENTREPRENEURSHIP}

empirical work in entrepreneurship could also be useful to researchers in financial risk management. Conversely, knowledge from financial risk management about biases and how to resolve those risks would be relevant to entrepreneurship, and entrepreneurial [corporate] finance in particular.

\section{Future Experimental Work}

Six interesting lines of future experimental research could build on the theoretical contributions of this paper. First, empirical work is encouraged to provide further support for the inclusion of overconfidence in the conceptual model of Figure 1. Deligonul et al. (2008) provide an argument that supports including overconfidence; however, an explanation by Hogarth and Karelaia (2012) suggests it should be removed.

Second, experiments could be performed to determine whether any of the factors that are known to influence risk attitudes also influence ambiguity attitudes and should be added to the conceptual model of Figure 1.

Third, empirical work is encouraged to better understand the multiple mechanisms mediating or moderating ambiguity attitudes in entrepreneurship. For instance, interaction effects between element of the ambiguity profile are anticipated (e.g., Camerer \& Weber, 1992), and above we identify gaps in experimental entrepreneurship to understand some of these anticipated effects. Experiments might be performed to address these gaps by studying the effect of simultaneously manipulating various elements of the ambiguity profile in ways that are representative of ambiguous situations in entrepreneurship. Also, researchers may propose and test for additional factors that might influence ambiguity attitudes, or for additional elements to be added to the ambiguity profile.

Fourth, the conceptual model presented here is based on experiments performed at the individual unit of analysis. The elements of the ambiguity profile closely resemble those contributing to Milliken's (1987) "effect uncertainty", or the perceived ambiguity an organizational administrator has regarding an environment's impact on their organization. This suggests that the conceptual model has the potential to be generalized to experimentally study ambiguity attitudes at the level of analysis of the firm.

Fifth, experiments could be performed to study whether this conceptual model is generalizable to ambiguous situations with outcomes that are not financial. For instance, experiments could be performed to study whether social or environmental outcomes influence ambiguity attitudes in the same way as do financial outcomes. These types of non-financial outcomes are important in social entrepreneurship and sustainable entrepreneurship. Sixth, further research could explore how ambiguities are tackled by successful start-ups. Specifically, how successful entrepreneurs use their limited resources to reduce ambiguities by gathering information. Lack of information contributes to the ambiguous situation that entrepreneurs face. Regardless of this lack of information, an entrepreneur must make decisions and typically must make those decisions quickly. For instance, in the pursuit of maintaining a positive cash balance, an entrepreneur needs to make many critical financial decisions under conditions of financial ambiguity. The ability to allocate resources to gather information to reduce ambiguity is an important resource allocation problem because an entrepreneur has 
limited resources (e.g., time; money, and expertise) to deploy. Consequently, which ambiguities the entrepreneur chooses to resolve and how they resolve those ambiguities, given their limited resources, makes for interesting future research questions. Choices between gambles using protocols from experimental research such as pairwise choice might provide information about entrepreneurs' preferences in resolving this ambiguity. Experimental work of this type would help inform our understanding of the entrepreneurial process, particularly resource allocation in entrepreneurship.

\section{Conclusion}

While a large body of work has been done on decision-making behaviors when faced with financial risk and uncertainty, relatively none has been done when faced with financial ambiguity. An entrepreneur routinely makes risky financial decisions in a context that is fraught with ambiguity. To understand how people behave under condition of ambiguity, we rely on rigorous findings from behavioral economics about ambiguity attitudes. These findings provide enough detail to build a conceptual model of experimental variables to consider in the study of ambiguity attitudes in a context that is representative of entrepreneurship, and a novel structure called the ambiguity profile that describes an ambiguous situation as perceived by the decision maker. Further, this paper clarifies terminology from various literature to encourage further interdisciplinary contributions. The contributions of this paper are useful to designing experiments that might add to interdisciplinary knowledge about decision-making behavior under condition of ambiguity, and during the entrepreneurial process in particular.

\section{Acknowledgements}

The authors thank Jane Russwurm and Katelyn Cousteils of the Writing and Communication Centre at the University of Waterloo, and we gratefully acknowledge the research funding from Social Sciences and Humanities Research Council (Canada) Insight Grant number 435-2014-2061 and the University of Waterloo. 


\section{References}

Blankenstein, N. E., Peper, J. S., Crone, E. A., \& van Duijvenvoorde, A. C. K. (2017). Neural mechanisms underlying risk and ambiguity attitudes. Journal of Cognitive Neuroscience, 29(11), 1845-1859.

Brunborg, G. S., Johnsen, B. H., Pallesen, S., Molde, H., Mentzoni, R. A., \& Myrseth, H. (2010). The relationship between aversive conditioning and risk-avoidance in gambling. Journal of Gambling Studies, 26(4), 545-559.

Budescu, D. V., Kuhn, K. M., Kramer, K. M., \& Johnson, T. R. (2002). Modeling certainty equivalents for imprecise gambles. Organizational Behavior and Human Decision Processes, 88(2), 748-768.

Camerer, C., Loewenstein, G., \& Prelec, D. (2005). Neuroeconomics: How neuroscience can inform economics. Journal of Economic Literature, 43(1), 9-64.

Camerer, C., \& Weber, M. (1992). Recent developments in modeling preferences: Uncertainty and ambiguity. Journal of Risk and Uncertainty, 5(4), 325-370.

Caplin, A., \& Leahy, J. (2001). Psychological expected utility theory and anticipatory feelings. The Quarterly Journal of Economics, 116(1), 55-79.

Cassar, G. (2014). Industry and startup experience on entrepreneur forecast performance in new firms. Journal of Business Venturing, 29(1), 137-151.

Curley, S. P., Yates, J. F. \& Abrams, R. A. (1986). Psychological sources of ambiguity avoidance. Organizational Behavior and Human Decision Processes, 38(2), 230-256.

Diebold, F. X., Doherty, N. A., \& Herring, R. P., N.J. (2010). The known, the unknown, and the unknowable in financial risk management: Measurement and theory advancing practice. Princeton University Press.

Deligonul, Z. S., Hult, G. T. M., \& Cavusgil, S. T. (2008). Entrepreneuring as a puzzle: An attempt to its explanation with truncation of subjective probability distribution of prospects. Strategic Entrepreneurship Journal, 2(2), 155-167.

Dew, N., Sarasathy, S., Read, S., \& Wiltbank, R. (2009). Affordable loss: Behavioral economic aspects of the plunge decision. Strategic Entrepreneurship Journal, 3(2), 105-126.

Du, N., \& Budescu, D. V. (2005). The effects of imprecise probabilities and outcomes in evaluating investment options. Management Science, 51(12), 1791-1803.

Du, N., Budescu, D. V., Shelly, M. K., \& Omer, T. C. (2011). The appeal of vague financial forecasts. Organizational Behavior and Human Decision Processes, 114(2), 179-189.

Forlani, D., \& Mullins, J. W. (2000). Perceived risks and choices in entrepreneurs' new venture decisions. Journal of Business Venturing, 15(4), 305-322.

Frisch, D., \& Baron, J. (1988). Ambiguity and rationality. Journal of Behavioral Decision Making, 1(3), 149-157.

Frishammar, J., Floren, H., \& Wincent, J. (2011). Beyond managing uncertainty: Insights from studying equivocality in the fuzzy front end of product and process innovation projects. IEEE Transactions on Engineering Management, 58(3), 551-563.

Galbraith, J.R., (1973). Designing complex organizations. Addison-Wesley.

Galbraith, J.R., (1974). Organization design: An information processing view. Interfaces, 4(3), $28-36$.

Grégoire, D. A., Cornelissen, J., Dimov, D., \& Burg, E. (2015). The mind in the middle: Taking stock of affect and cognition research in entrepreneurship. International Journal of Management Reviews, 17(2), 125-142. 
Heath, C., \& Tversky, A. (1991). Preference and belief: Ambiguity and competence in choice under uncertainty. Journal of Risk and Uncertainty, 4(1), 5-28.

Hogarth, R. M., \& Karelaia, N. (2012). Entrepreneurial success and failure: Confidence and fallible judgement. Organization Science, 23(6), 1733-1747.

Hsu, D. K., Simmons, S. A., \& Wieland, A. M. (2017). Designing entrepreneurship experiments. Organizational Research Methods, 20(3), 379-412.

Hsu, M., Bhatt, M., Adolphs, R., Tranel, D., \& Camerer, C. F. (2005). Neural systems responding to degrees of uncertainty in human decision-making. Science, 310(5754), 1680-1683.

Huettel, S. A., Stowe, C. J., Gordon, E. M., Warner, B. T., \& Platt, M. L. (2006). Neural signatures of economic preferences for risk and ambiguity. Neuron, 49 (March 2, 2006), 765-775.

Knight, F. H. (1921). Risk, uncertainty and profit. Houghton Mifflin.

Kollmann, T., Stöckmann, C., \& Kensbock, J. M. (2017). Fear of failure as a mediator of the relationship between obstacles and nascent entrepreneurial activity-An experimental approach. Journal of Business Venturing, 32(3), 280-301.

Krain, A. L., Wilson, A. M., Arbuckle, R., Castellanos, F. X., \& Milham, M. P. (2006). Distinct neural mechanisms of risk and ambiguity: A meta-analysis of decision-making. Neuroimage, 32(1), 477-484.

Kunreuther, H., Meszaros, J., Hogarth, R. M., \& Spranca, M. (1995). Ambiguity and underwriter decision processes. Journal of Economic Behavior \& Organization, 26(3), 337-352.

LeDoux, J. E. (1996). The emotional brain: The mysterious underpinnings of emotional life. Simon and Schuster.

Lerner, J. S., \& Keltner, D. (2000). Beyond valence: Toward a model of emotion-specific influences on judgement and choice. Cognition \& Emotion, 14(4), 473-493.

Lévesque, M., \& Schade, C. (2005). Intuitive optimizing: Experimental findings on time allocation decisions with newly formed ventures. Journal of Business Venturing, 20(3), 313-342.

Liu, Y., \& Öncüler, A. (2017). Ambiguity attitudes over time. Journal of Behavioral Decision Making, 30(1), 80-88.

March, J. G., \& Shapira, Z. (1992). Variable risk preferences and the focus of attention. Psychological Review, 99(1), 172-183.

McMullen, J. S., \& Shepherd, D. A. (2006). Entrepreneurial action and the role of uncertainty in the theory of the entrepreneur. Academy of Management Review, 31(1), 132-152.

Onay, S., La-ornual, D., \& Öncüler, A. (2013). The effect of temporal distance on attitudes toward imprecise probabilities and imprecise outcomes. Journal of Behavioral Decision Making, 26(4), 362-374.

Platt, M. L., \& Huettel, S. A. (2008). Risky business: The neuroeconomics of decision making under uncertainty. Nature Neuroscience, 11(4), 398.

Rustichini, A., Dickhaut, J., Ghirardato, P., Smith, K., \& Pardo, J. V. (2005). A brain imaging study of the choice procedure. Games and Economic Behavior, 52(2), 257-282.

Sarasvathy SD. (2001). Effectual reasoning in entrepreneurial decision making: Existence and bounds. Academy of Management Best Paper Proceedings ENT D1-D6.

Shapira, Z. (1995). Risk taking: A managerial perspective. Russell Sage.

Schoemaker, P. J. H. (1989). Preferences for information on probabilities versus prizes: The role of risk-taking attitudes. Journal of Risk and Uncertainty, 2(1), 37-60.

Schwarzkopf, D. L. (2006). Stakeholder perspectives and business risk perception. Journal of Business Ethics, 64(4), 327-342. 
Shepherd, D. A., Williams, T. A., \& Patzelt, H. (2015). Thinking about entrepreneurial decision making. Journal of Management, 41(1), 11-46.

Smith, K., Dickhaut, J., McCabe, K., \& Pardo, J. (2002). Neuronal substrates for choice under ambiguity, risk, gains, and losses. Management Science, 48(6), 711-718.

St Andre, J., \& Reilly, S. (2007). Effects of central and basolateral amygdala lesions on conditioned taste aversion and latent inhibition. Behavioral Neuroscience, 121(1), 90-99.

Sturm, V. E., Haase, C. M., \& Levenson, R. W. (2016). Emotional dysfunction in psychopathology and neuropathology: neural and genetic pathways. genomics, circuits, and pathways in clinical neuropsychiatry. In L Thomas (Eds). San Diego, CA: Elsevier Academic Press.

Sykes, H. B., \& Dunham, D. (1995). Critical assumption planning: A practical tool for managing business development risk. Journal of Business Venturing, 10(6), 413-424.

Tanaka, Y., Fujino, J., Ideno, T., Okubo, S., Takemura, K., Miyata, J., Kawada, R., Fujimoto, S., Kubota, M., Sasamoto, A., Hirose, K., Takeuchi, H., Fukuyama, H., Murai T., \& Takahashi, H. (2015). Are ambiguity aversion and ambiguity intolerance identical? A neuroeconomics investigation. Frontiers in Psychology, 5(1550).

Trautmann, S. T., Vieider, F. M., \& Wakker, P. P. (2008). Causes of ambiguity aversion: Known versus unknown preferences. Journal of Risk and Uncertainty, 36(3), 225-243.

Yates, J. F., \& Stone, E. R. (1992). The risk construct. In Frank Yates, J. (Eds.). Risk taking behavior. Wiley.

Zhang, S. X., \& Cueto, J. (2017). The study of bias in entrepreneurship. Entrepreneurship Theory and Practice, 41(3), 419-454. 